\title{
MRI Finding in Delayed Extensive Brain Lesions after Radiation Therapy: Cortical Laminar Necrosis and White Matter Myelinolysis
}

\author{
Yeting Zhou' ${ }^{1}$, Guangsheng Wang ${ }^{2}$, Xiaodong Chen ${ }^{2}$, Daoming Tong ${ }^{2}$ \\ ${ }^{1}$ Medical Evaluation Unit, Shuyang People'Hospital, Shuyang, China \\ ${ }^{2}$ Departments of Neurology, Shuyang People’Hospital, Shuyang, China \\ Email: tongdaoming@163.com
}

Received September 11, 2011; revised October 13, 2011; accepted November 2, 2011

\begin{abstract}
The focal and diffuse cerebral white matter injury can be caused by treatment with radiation therapy for cranial tumors. However, the literature rarely describes a MRI finding in radiation-induced delayed extensive cerebral injury. Our objective was to report a rare case who had a delayed extensive hyperintensity injury in brain on MRI after radiation therapy due to nasopharyngeal cancer. A MRI was performed on a 40-year-old patient with extensive brain damage who had the radiation therapy two years ago. MRI finding was evaluated. On MRI, T2-weighted MRI showed an extensive hyperintensity after treated by irradiation. The radiographic pattern of extensive cerebral injury is relatively distinct. It involves the white matter and gray matter in cerebral, cerebellum, medulla oblongata, pons, internal capsule and thalamus bilaterally. Our observations demonstrate that the extensive hyperintensity lesions in brain on MRI after radiation therapy is a cortical laminar necrosis and white matter myelinolysis.
\end{abstract}

Keywords: Radiation-Induced Brain Damage; Magnetic Resonance Imaging; Encephalopathy; Cortical Laminar Necrosis; Demyelination

\section{Introduction}

Radiation therapy can prolong survival of cancer patients to improve their quality of life, but it occasionally induces the human body, including brain radiation injury. Radiation-induced brain damage is considered an uncommon delayed complication among cancer survivors who have undergone whole brain irradiation for brain tumors [1-5]. However, the extensive damages after brain radiotherapy, especially in extensive bilateral brain, cerebellum and brain stem damage, bilateral internal capsule and thalamus represent a rare late-onset events. In this paper we report a nasopharyngeal carcinoma patient who had a rare delayed complication of a wide range of brain damage after radiotherapy and his brain magnetic resonance (MR) imaging finding.

\section{Meterial and Methods}

A 40-year-old male patient, he had suffered resection and radiation therapy because of the nasopharyngeal carcinoma for 2 years. He was symptom-free until 1 month before admission at which time he presented with symptoms of bilateral progressive weakness to complete disability, slurred speech, and slightly dysphagia.
At the age of 38 years, he had a series of episodes of "nasopharyngeal carcinoma, auricle inflammation" and he was begun treatment with radiation therapy such as 70 Gy nasopharyngeal and gamma knife 8 Gy irradiation in hospital, the patient's symptoms slowly recovered and he was administered. The temperature was $37.3^{\circ} \mathrm{C}$, the pulse 94, and the respirations 22 . The blood pressure was 130/90 mmHg. A general physical examination revealed no important abnormalities. On neurologic examination, the patient was fully alert and oriented; his speech was slurred. He had four limbs palsy with grade 3-4 in the upper limbs and grade 3 in the lower limbs, the sensory impairment was not demonstrated. The deep tendon reflexes of the extremities were disappeared. The bilateral Hoffmann's sign and Babinski's sign were present, but decline in cognitive faculties. The cranial-nerve functions were normal. On admission, a brain CT scan, brain MRI, and the others examination were performed .Brain MRI finding, pathogenesis and prognosis were then analyzed.

\section{Result}

Brain CT scan showed postoperative changes. Brain T2weighted cranial magnetic resonance imaging (MRI) 
showed an extensive high signal intensity in the bilateral cerebellar hemispheres and the medulla oblongata (Figures 1(a) and 1(b)), the bilateral cerebellum and pons (Figure 1(c)), the pons (Figure 1(d)), the bilateral temporal cortex, internal capsule and thalamus (Figure 1(e)), the bilateral frontal and temporal cortex, internal capsule and thalamus (Figure 1(f)).

On the second day's admission, serum glucose, electrolytes was at normal levels. Low-density lipoprotein was slightly increased. Blood chemistry analysis and other toxic substances in the serum screening results were negative. Bultrasound on liver, gallbladder, spleen, pancreas was no abnormal. A chest radiograph that the heart and lungs were normal. On the three days after admission, a lumbar puncture was performed and CSF examination was normal. An electroencephalogram was normal.

After admission, the diagnosis of radiation-induced delayed extensive brain damage was made and the treatment with corticosteroid was begun. His condition was improved, and he was discharged home with walking a little unstable. Unfortunately, the patient was lost to further follow-up.

\section{Conclusions}

Radiation-induced local brain damage after radiation of nasopharyngeal carcinoma have been reported [1-5], the white-matter changes are always bilateral ultimately. Local radiation injury is commonly seen in the temporal lobe injury on MR [1]. Diffuse radiation injury is characterized by periventricular increased signal on proton-density and T2-weighted MR images [5]. White matter damage caused by radiation therapy has also been confirmed by animal experiments [6]. However, delayed extensive brain damage after brain radiation is still less report. Our present this case, the brain damage on brain MRI is extreme extensive, including the extensive high signal intensity change in the bilateral frontal and temporal cortex, bilateral cerebellar hemispheres and the medulla oblongata, upper pons and lower pons, and bilateral internal capsule and thalamus. The patient had no prior episode of ischemia, hypoxia or exposure to carbon monoxide, so hypoxic ischemic encephalopathy was excluded. On admission, his blood glucose a levels were in normal range, hypoglycemic encephalopathy can also be excluded. Our patient had a history of nasopharyngeal

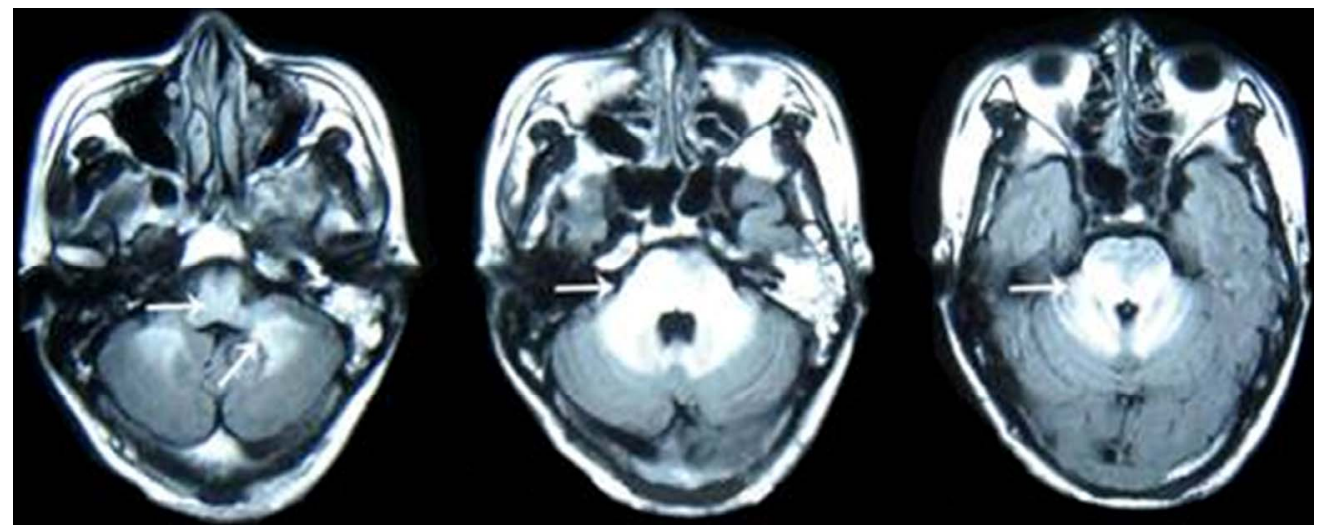

(a)

(b)

(c)

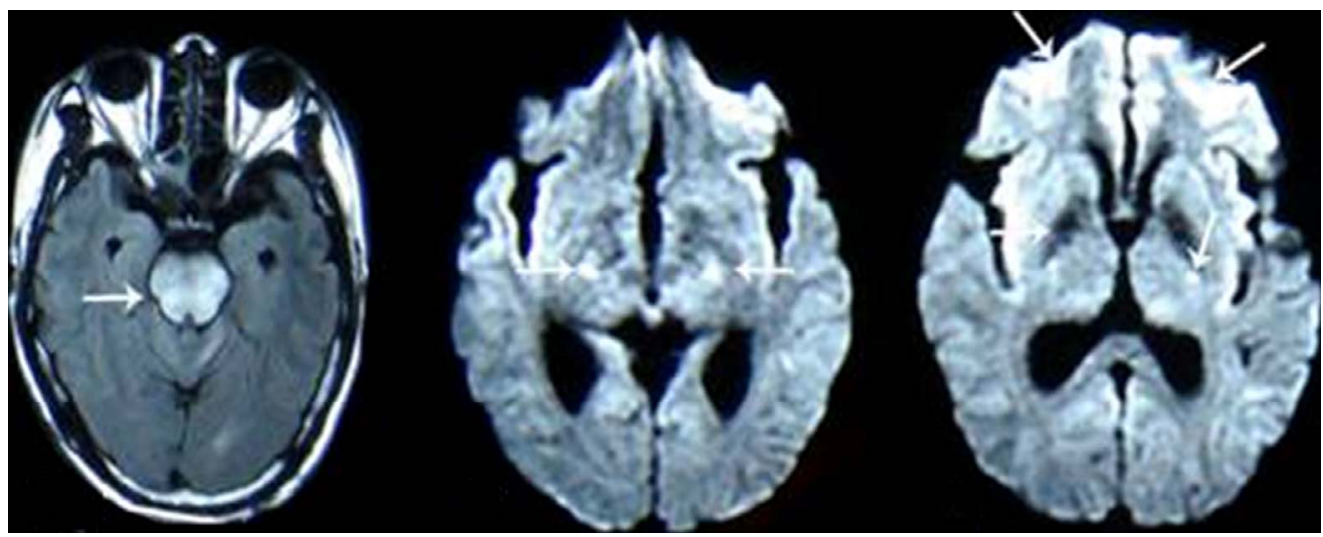

(d)

(e)

(f)

Figure 1. (a) T2-weighted images show high signal intensity in this patient. (b) Bilateral cerebellar hemispheres and medulla oblongata. (c) Bilateral cerebellar and pons. (d) Pons. (e) Bilateral temporal cortex, internal capsule and thalamus. (f) Bilateral frontal cortex, temporal cortex, internal capsule and thalamus. 
cancer and ear infection, and his symptoms was relieved when he was undergone a surgery and radiation therapy of nasopharyngeal carcinoma in two years ago. In addtion, CT scan of the nasopharyngeal and systemic examination had no evidence of recurrence or metastasis of cancer on admission, the diagnosis of radiation-induced delayed extensive brain damage had been made.

Although our patient had an extensive brain damage, especially the extensive high signal change in the pons, the patient is neither the clinical manifestations of the network of nuclei and interconnecting tracts damages in the upper brain stem, nor the clinical signs of brain nuclei damage in the upper pons. Despite patient had a high signal changes in the cortex, he did not diminish the clinical manifestations of intelligence. This shows that the patient's brain damage is mainly in white matter, while less in gray matter. Prior study had confirmed that it is a radiation leukoencephalopathy [7]. Radiation leukoencephalopathy is not very common a complication after radiotherapy. The mechanism of the delayed brain damage after radiation therapy has not yet reached a complete consensus. Most studies considered that the radiation therapy-induced delayed brain damage is associated with the demyelination and necrosis [2,7,8]. It has also been confirmed by histopathology [7]. In some instances, the delayed brain damage is associated with ischemia or vascular occlusion [7,9]. Recent, other suggests that these high signals represent neuronal apoptosis [10]. High signals in the white matter on MRI represent a demyelination which has been largely accepted, but we considered that the high signals in the cortex may represent a cortical laminar necrosis. Therefore, the radiation therapyinduced delayed extensive brain damage is a relatively rare cortical laminar necrosis and white matter myelinolysis. On the other hand, MRI is not only helpful for diagnosis, but also to predict the prognosis of patient. In most cases, delayed leukoencephalopathy after radiation therapy have been improved or relieved [1]. Patient with the formation of cyst may cause a poor functional recovery [1]. Rarely, the patient with dementia often develops a poor prognosis, even death [11]. This shows that the prognosis of delayed radioactive brain damage often related with the severity of brain lesions. In our case, MRI showed high signal although more extensive distribution, but not very serious. The outcome of patient has been improved by continuous use of corticosteroid treatment. This suggests that these high signals not only represent a cortical laminar necrosis and white matter myelinolysis, but also confirm that the radiation therapy-induced corti- cal laminar necrosis and white matter myelinolysis is not completely irreversible.

\section{REFERENCES}

[1] Y. X. Wang, A. D. King, H. Zhou, et al., "Evolution of Radiation-Induced Brain Injury: MR Imaging-Based Study,” Radiology, Vol. 254, No. 1, 2010, pp. 210-218. doi:10.1148/radiol.09090428

[2] J. S. Tsuruda, K. E. Kortman, W. G. Bradley, et al., "Radiation Effects on Cerebral White Matter: MR Evaluation," American Journal of Roentgenology, Vol. 149, No. 1, 1987, pp. 165-171.

[3] Y. I. Chan, S. F. Leung, A. D. King, et al., "Late Radiation Injury to the Temporal Lobes: Morphologic Evaluation at MR Imaging,” Radiology, Vol. 213, No. 3, 1999, pp. 800-807.

[4] A. Asai and K. Kawamoto, "Radiation-Induced Brain Injury,” Brain Nerve, Vol. 60, No. 2, 2008, pp. 123-129.

[5] P. L. Khong, D. L. Kwong, G. C. Chan, et al., "Diffusion-Tensor Imaging for the Detection and Quantification of Treatment-Induced White Matter Injury in Children with Medulloblastoma: A Pilot Study,” American Journal of Neuroradiology, Vol. 24, No. 4, 2003, pp. 734-740.

[6] S. Wang, E. X. Wu, D. Qiu, et al., "Longitudinal Diffusion Tensor Magnetic Resonance Imaging Study of Radiation-Induced White Matter Damage in a Rat Model,” Cancer Research, Vol. 69, No. 3, 2009, pp. 1190-1198.

[7] P. E. Valk and W. P. Dillon, "Radiation Injury of the Brain,” American Journal of Neuroradiology, Vol. 12, No. 1, 1991, pp. 45-62.

[8] M. Becker, G. Schroth, P. Zbären, et al., "Long-Term Changes Induced by High-Dose Irradiation of the Head and Neck Region: Imaging Findings," Radiographics, Vol. 17, No. 1, 1997, pp. 5-26.

[9] A. Muthukrishnan, M. Bajoghli and J. M. Mountz, "Delayed Development of Radiation Vasculopathy of the Brain Stem Confirmed by F-18 FDG PET in a Case of Anaplastic Astrocytoma,” Clinical Nuclear Medicine, Vol. 32, No. 7, 2007, pp. 527-531. doi:10.1097/RLU.0b013e31806469ef

[10] K. Sano, K. Morii, Sato M, et al., "Radiation-Induced Diffuse Brain Injury in the Neonatal Rat Model-Radiation-Induced Apoptosis of Oligodendrocytes,” Neurologia Medico-Chirurgica (Tokyo), Vol. 40, No. 10, 2000, pp. 495-499. doi:10.2176/nmc.40.495

[11] M. C. Vigliani, C. Duyckaerts, J. J. Hauw, et al., "Dementia Following Treatment of Brain Tumors with Radiotherapy Administered Alone or in Combination with Nitrosourea-Based Chemotherapy: A Clinical and Pathological Study," Journal of Neuro-Oncology, Vol. 41, No. 2, 1999, pp. 137-149. doi:10.1023/A:1006183730847 